\title{
Modelling Local Patterns of Children Mortality. A Bayesian Spatio-Temporal Analysis.
}

\author{
Alejandro Lome-Hurtado ( $\square$ alejandro.lomehu@anahuac.mx ) \\ Universidad Anahuac Mexico https://orcid.org/0000-0003-1241-4553 \\ Jacques Lartigue Mendoza \\ Universidad Anahuac Mexico \\ Juan Carlos Trujillo \\ University of York
}

\section{Research article}

Keywords: Children health, Bayesian mapping, high-risk areas, space-time interactions, Mexico

Posted Date: July 25th, 2019

DOI: https://doi.org/10.21203/rs.2.11961/v1

License: (1) This work is licensed under a Creative Commons Attribution 4.0 International License.

Read Full License

Version of Record: A version of this preprint was published at BMC Public Health on January 6th, 2021.

See the published version at https://doi.org/10.1186/s12889-020-10016-9. 


\section{Abstract}

Background: The number of death children at the international scale are still high, but with proper spatially-targeted health public policies this number could be reduced. In Mexico, children mortality is a particular health concern due to its alarming rate all throughout North America. The aims of this study are i) to model the change of children mortality risk at the municipality level, (ii) to identify municipalities with high, medium and low risk over time and (iii) to ascertain potential high-risk municipalities across time, using local trends of each municipality in Greater Mexico City. Methods: The study uses Bayesian spatiotemporal analysis to control for space-time patterns of data. This allow to model the geographical variation of the municipalities within the time span studied. Results: The analysis shows that most of the high-risk municipalities are in the north, west, and some in the east; some of such municipalities show an increasing children mortality risk over time. The outcomes highlight some municipalities which show a medium risk currently but are likely to become high risk along the study period. Finally, the odds of children mortality risk illustrate a decreasing tendency over the 7-year framework. Conclusions: Identification of high-risk municipalities may provide a useful input to policy-makers seeking out to reduce the incidence of children mortality, since it would provide evidence to support geographical targeting for policy interventions.

\section{Introduction}

There is a public concern regarding the high percentage of mortality on children. At the international scale, there were 5.6 million deaths of children in the year 2016 [1]. Therefore, an increasing policy interest in improving children's health has been witnessed, which is reflected in the United Nations' third Sustainable Development Goal (SDG) on good health and wellbeing, and particularly in the SDG 3 target to end preventable deaths of new-born and children under five by 2030 [2].

In Mexico, the children mortality rate under 5 years old decreased from $17.3 \%$ to $13.4 \%$ for 2010 and 2017, respectively. However, these numbers are still higher than those observed in developed countries. For instance, these rates were 7.3 and 6.6 in the same years in the United States [3]. Similarly, the probability of dying at age 5-14 years (per 1,000 children age 5) in Mexico were 2.8 and 2.5 for the years 2010 and 2017, respectively. Meanwhile, in the United States this figure was 1.3 in the same period [3], [4]. According to recent official data (authors' elaboration based on [5]), child mortality in Mexico has seen a reduction, passing from a rate of 1,200 to 800 deaths per 1,000 live children from 2011 to 2017, respectively. Nevertheless, the rate is still above of the Sustainable Development Goal target of 20 deaths per 1,000 live births.

This paper focuses on modelling children mortality risk trends across different geographical areas. There has been an increasing interest in recent years to take into account the modelling of the geographical dimension of children mortality risk [6]-[9]. Gayawan et al. [6] illustrated the regional variations on children mortality among 10 West African countries. They found some clusters of higher child mortality in northwest and northeast of Nigeria. Jimenez-Soto et al. [7] showed the disparities in child mortality 
across rural-urban locations and regions in Cambodia. In Papua, New Guinea, a study found important child mortality disparities between rural and urban populations and variations across inter and intra regions [8]. In Mexico, a similar study [10] analyzed the trend on children mortality caused by diarrhoea in all Mexican states. They identified different spatial patterns across time on the peak mortality rate for the different states.

However, there is a need of analyzing not just the spatial pattern but also the local trend of each geographical unit and identify their level of risk over time, which may allow to gain better understanding. The importance of accounting for the potential spatial structure on the data is that communities are often clustered with respect to their socioeconomic background [11]. Hence, it is likely that people with higher socioeconomic status live close to each other. This may also apply for people with lower socioeconomic status. Socioeconomic status may also vary across time for both individuals and neighbourhoods, with important influences on health risk [11]. Therefore, children mortality may not only vary over space, but also over the time, as it has been the case in other health studies; that is the case of the spatio-temporal variation of stomach cancer risk [12], and asthma risk [13]. People's health risk may vary over space and time due to changes in health-related behaviours such as physical activity, smoking, and diet [14]. Thus, there is a need of analyzing not only the spatial pattern but also the local trend of each geographical unit and identify their corresponding level of risk over time.

The aims of this study are to (i) to model the change of children mortality risk at the municipality level, (ii) to identify municipalities with high, medium and low risk over time and (iii) to explore potential high-risk municipalities across time, using local trends of each municipality. Locations with high children mortality risk should be a priority for policy intervention, so this analysis may provide important baseline information for decision-makers. The identification of spatial and temporal trends across different areas may provide important inputs for decision-makers in designing programmes to tackle health inequalities [15]. Programmes, which are spatially targeted, may focus on small locations, allowing the policy measures to have a more effective local impact. Vulnerable and local groups are more benefited in such cases compared with other programmes, where the resources are not directed towards specific geographical areas [16], [17]. This study uses a Bayesian modelling approach [18], due to the space, time, and space-time structure of the data. This Bayesian methodology is based on random effects which allow to model the geographical variation of children mortality over time. This approach was used in the area of criminology [19] and up to our knowledge, this is the first time used to account for the space-time structure on children mortality risk.

\section{Data And Methods}

\subsection{Area of study and Children mortality data}

Greater Mexico City, one the most populated urban areas in the world, is the third largest metropolis in OECD countries, and the world's largest outside Asia [20]. It consists of 16 municipalities within Mexico City and 59 municipalities of the State of Mexico. It had 20,892,724 inhabitants in 2015, according to the 
Mexican National Institute of Statistics and Geography (INEGI) [21], with a land area of 7,866 square kilometers. It is considered the most important metropolitan area in Mexico in terms of the economy, producing $23 \%$ of the country's gross domestic product in 2010 [20]. The study explored the catalog of death records of the Mexican Ministry of Health in the municipalities of Greater Mexico, 75 municipalities, from January 2011 to December 2017. The total number of death records were $n=79,4401$ and among them there were 39,263 dead children in the region, in the study period. This information was aggregated in order to perform a spatial and temporal analysis, at the municipality level. Children were considered from 0 to 12 years old [22].

Global Moran Index [23] and Autocorrelation Function (ACF) statistical tests were carried out in order to analyze the potential presence of spatial autocorrelation and serial correlation of the data, respectively. The Global Moran Index of the data for each year was positive and significant with a mean value of 0.32 and a $p$ value $<0.0001$, this illustrates the presence of positive spatial autocorrelation in the records. This result showed the existence of some nearby municipalities with similar mortality risk. The ACF mean was 0.58 (lagged 1 year for each municipality), across all the municipalities. This number illustrated the evidence of serial correlation, the association on a certain level of the observed mortality data across time.

\subsection{Statistical analysis}

To model the potential spatial, temporal and spatio-temporal structure of the children mortality data, this study uses Bayesian analysis. For modelling the probability of having a child or not, the dead analysis assumes a Binomial likelihood [24], yit Binomial (nit,pit). Specifically, let yit be the number of cases of dead children in each municipality $i$ at the time point $t$ ( $=2012,2013 . .2017)$; nit represents the number of children in the municipality $i$; and $\mu$ it denotes children mortality risk. According to Law et al. [25] and Li et al. [19] the children mortality risk can be modelled as:

logit $\mu i t=a+s i+u i+d 0 t^{\star}+v t+d 1 i^{\star}+\varepsilon i t(1)$

where $\mathrm{a}$ is the overall logit children mortality risk across the 6-year period; the terms si and ui capture the spatial structure of the data. This component is common over the study period. In turn, $\mathrm{d} 0 \mathrm{t}^{\star}+\mathrm{vt}$ are the overall linear time trend to all the municipalities with additional Gaussian noise (vt) that allows for nonlinearity in the overall trend pattern, vt has the following distribution vt $\sim N(0, \sigma v 2)$. The following expression, $\mathrm{d} 1 \mathrm{it}{ }^{\star}$, denotes the spatial-temporal structure of the data which allows each municipality to have its own trend. Therefore, this component represents and assumes a linear departure of the local temporal trend of each municipality from the common trend; such local trend can be increasing, decreasing or a stable tendency from the overall linear pattern. Finally, $\varepsilon i t \sim N(\sigma \varepsilon)$ is the component for the variability that is not explained by the other terms. This may include overdispersion, that is, when the variation of the data is higher than its mean.

We assigned the BYM (Besag, York and Mollié) model [26] to the spatial component (si+ui). The spatial component is composed by si and ui, which represents the spatially structured and unstructured random 
effects, respectively. The last term follows a Gaussian distribution, whereas si follows an intrinsic conditional autoregressive (ICAR) prior model. Thus, si includes a spatial adjacency matrix W of size N X $\mathrm{N}$, where the diagonal values are wij $=1$ if municipalities $\mathrm{i}$ and $\mathrm{j}$ share a common boundary, otherwise wij $=0$. The ICAR prior on the spatial random effect considers the potential spatial autocorrelation, neighbouring areas are more likely to have similar values. In our specific case, it means that nearby municipalities are assumed to have similar mortality children risk rates. The same BYM is allocated to d1 i. In this case, nearby municipalities would have similar changes or local trends of mortality children risk compared to those that are far apart. This component may also control for the potential endogeneity due to the interaction between space and time. Finally, all random effect standard deviations such as ov2 and $\sigma \epsilon$ have a positive half Gaussian prior $\mathrm{N}+\infty(0,10)$ following the Gelman $(2006)$ criterion.

To classify each municipality into hot, cold and neither-hot-nor-cold spots (high, low and medium risk) across time, I used the values of the posterior probability of the spatial component pexpui+si>1data). Thus, the values greater than 0.8 , between 0.2 and 0.8 , and lower than 0.2 were classified as hot, neitherhot-nor-cold spots and cold spots (high, low and medium risk municipalities), respectively. This criterion has been used in previous studies [19], [27]. Note here that the component expui+si indicates the odds average risk of the study period for each municipality with respect to a (overall logit children mortality risk). Next, to measure the local trend of each classified municipality, we used the values of the posterior probability of the local slopes pd1i>0hi, data). This would allow to measure its local dynamic over the study period as increasing, stable and decreasing. Thus, the values greater than 0.8 , between 0.2 and 0.8 , and lower than 0.2 were classified as increasing, stable or decreasing trend compared with the overall trend for each municipality.

The model was implemented in R and WinBUGS (statistical software). We ran MCMC chains of 100,000 (for this number the model reaches convergence) with different initial values for the model. We used 70,000 iterations for the model for making inferences, after having burned in the first 30,000 . The convergence was examined by visual inspection of the history plots and through the Gelman-Rubin diagnostic [28]. The values from the Gelman-Rubin diagnostic remained lower than 1.04 for every single model parameter, showing that the chains have achieved convergence after the burn-in period for the model.

\section{Results}

\subsection{Descriptive Analysis}

Table 1 provides an overview of the descriptive statistics of the observed children mortality risk in Greater Mexico. Overall, there is a slight decrease of the average of the observed children mortality risk per 100 inhabitants during these years $(8.17,6.54$, and 5.41 in 2011, 2014 and 2017, respectively).

Insert Table 1 
Figure 1 illustrates the temporal evolution of the observed children mortality risk by municipality in Greater Mexico City, at different time points throughout the study period 2011, 2014, and 2017 (start, middle, and end). The green and red colours show the lower and higher risk, respectively. Overall, municipalities with higher risk are in the north, partially in the west, and in the east. Conversely, there is a clear cluster (stronger green colour) located in the centre-west which has the lowest mortality risk.

\section{Insert Figure 1}

\subsection{Modelling spatial-temporal patterns}

Figure 2a illustrates the odds risk of child mortality in each municipality compared to the average over the studied period. An odd risk value that is above or below 1 suggests a higher or a lower risk respectively for this municipality compared to the Greater Mexico City average across the 7 years. The figures show mostly that areas in the north, west and few in the east, in general the surroundings municipalities of Mexico City, are characterized by having higher risks of mortality of children, whilst, broadly, Mexico City's municipalities and few in the north part have lower risks of child mortality. Figure $2 \mathrm{~b}$ illustrates the overall time trend of relative risk, compared to the Greater Mexico City average from 2011 to 2017. Overall, there is a slight decreasing tendency of such risk.

\section{Insert Figures $2 a$ and $2 b$}

Figure 3a, 3b, and 3c show the hot spots, neither-hot-nor-cold spots, and cold spots of child mortality risk of each municipality (high, medium, and low risk, respectively). There were 39 ( $50 \%$ of the total), 16 ( $21 \%$ of the total) and 20 ( $27 \%$ of the total) municipalities with high risk, medium, and low risk, respectively. In general, high risk municipalities (hot spots) are located in the north, west and east of the metropolitan area with a few spots in the south (see Figure 3a). Meanwhile, most of the low risk municipalities are located in Mexico City and its surrounding municipalities in the north-west area (Figure 3b). Finally, Figure $3 \mathrm{c}$ shows that the medium risk municipalities are mostly scattered in the north and south-west. All these classified municipalities are significant at the $95 \%$ credible interval $(\mathrm{Cl})$.

The inserted graphs in figures $3 a, 3 b$, and $3 c$ show the different trends of the observed risk (per 100 inhabitants; the black solid dots), the estimated risk (open circles and dashed line) with $95 \% \mathrm{Cl}$ (grey region), and the estimated common trend (black line) of such mortality risk over time.

\section{Insert Figure 3a}

Figure 3a shows that most of the high-risk municipalities (72\%) had a stable dynamic, whereas 6 of the high-risk municipalities (representing 15\%) had an increasing trend in children mortality risk. Leaving just 5 high-risk municipalities (13\%) with a decreasing trend over time. Figure $3 \mathrm{~b}$ shows the medium risk municipalities, of which $25 \%$ showed an increasing trend in risk. The majority of these medium risk municipalities presented a stable tendency $(50 \%)$; whereas, those municipalities with a decreasing trend represented the $25 \%$. 
Finally, Figure $3 \mathrm{c}$ illustrates that a few numbers of low risk municipalities, $15 \%$, mainly located in the downtown of Mexico City, showed a relative increase in children mortality risk over time. However, most of these low risk municipalities (65\%) had a stable trend in such period. Leaving just the $20 \%$ of municipalities, under this category, with a decreasing trend.

\section{Insert Figure $3 c$}

\section{Discussion}

This study has focused on the temporal dynamics of children mortality across municipalities in Greater Mexico City. It has identified the municipalities with high children mortality risk and those with medium risk that may become high risk municipalities as well. To the best of our knowledge, this is the first work in this area which has taken in consideration space, time, and space-time patterns using a Bayesian approach.

Around $50 \%$ of the high-risk municipalities were in the in the north, west, and in the east of Greater Mexico with a few spots in south. This is an expected result since the best economic and socioeconomic conditions are located in Mexico City, leaving the surrender municipalities with lower economic and social standing. According to Chandrashekhar et al [29] and Justice Moses et al [30], there is a positive association between deprived economic conditions and children mortality. It means that as lower the level of income is, higher the probabilities of having higher children mortality rates. The north, west and east of Greater Mexico are characterized by having a relatively lower socioeconomic and education levels than the average (see figure A2 in the Appendix). Six high-risk municipalities (Nextlalpan, Ozumba, Tenango del Aire, Tequixquiac, Tultepec and Tonanitla) presented an increasing trend over time, most of these municipalities are in the north area. Similarly, there were 6 medium risk municipalities (Apaxco, Chiconcuac, Teotihuacán and Tlalmanalco), representing $15 \%$ of the total medium risk municipalities, with an increasing trend over time. It means that these areas may become high risk municipalities in the short term. Such municipalities are located in the north of the Greater Mexico, excepting Tlalmanalco, facing also deprived economic and social conditions (see figure A2 in the Appendix). Finally, the odds mortality risk shows a decreasing trend over the 7-year period of study. This slight decreasing may be explained, partially, by the results of different public health policies. For instance, the Ministry of Health in Mexico has created a number of public programmes to decrease the neonatal mortality risk. These include 'Programa de Accion: Arranque Parejo en la Vida, 2002', 'Programa de Accion Especifico 20072012, 2008' and Programa de Accion Especifico Salud Maternal y Perinatal, 2013-2018' [31].

These results should be interpreted with caution due to some methodological limitations. We have assumed that there is not mobility of children. This assumption may not apply in a dynamic area as Greater Mexico City. However, it is not easier to account for the mobility of people which would require more precise data; therefore, we did not measure it as other studies [32]-[34]. However, key strengths of this study include the specific inclusions of time, space, and space-time structures, which are important to 
consider due to the nature of the data. Therefore, it is possible to derive more robust outcomes in the analysis.

\section{Conclusion}

The findings of this study, showing the evolution of high and medium risk municipalities, may provide an important input to decisions on policy to reduce children mortality risk. McLaughlin et al. [35] highlight the importance of robust data analysis and the knowledge of the local context as inputs for policy decisions. In the area of health, Ugarte et al. [36] illustrate that spatial and temporal trends provide useful information for addressing health inequalities. Therefore, the identification of municipalities at the highest risk of children mortality, especially those with an increasing trend over time (Nextlalpan Ozumba, Tenango del Aire, Tequixquiac, Tultepec and Tonanitla), would permit the geographical targeting of policy efforts to reduce it. This may offer significant benefits in terms of developing cost-effective policy, given the overall scarcity of healthcare resources in Mexico. In particular, the identification of current mediumrisk municipalities with an increasing trend over time could be important in developing more proactive geographically-targeted policy initiatives. Geographical targeting of policy may also bring benefits in enhancing the confidence and capacity of the local participation, which would in turn increase the impact of such policies [16][17].

\section{Declarations}

\section{Ethics approval and consent to participate}

Not applicable

Consent for publication

Not applicable

Availability of data and material

Data are freely available at:

http://www.dgis.salud.gob.mx/contenidos/basesdedatos/Datos_Abiertos_gobmx.html

Competing interests

The authors declare that they have no competing interests.

\section{Authors' contributions}

ALH developed the spatio-temporal statistical model and

performed the model programming, fitting and interpretation of 
the results. He drafted the sections in this paper.

All the authors contributed to the design of the study.

JCT and JLM contributed with the introduction,

discussion and conclusions. They wrote the final manuscript.

All authors read and approved the final manuscript.

\section{Funding}

No funding required

Author details

1Department of Business and Economics, Anahuac University, Mexico.

2 Environment and Geographical Department, University of York, England.

Ethics approval and consent to participate

Not applicable.

\section{References}

[1]D. You et al., "United Nations Inter-agency Group for Child Mortality Estimation (UN IGME). Global, regional, and national levels and trends in under- 5 mortality between 1990 and 2015, with scenariobased projections to 2030: a systematic analysis by the UN Inter-agency" Lancet, vol. 386, no. 10010, pp. 2275-2286, 2015.

[2]U. Nations, "Sustainable development goals," (n.d). [Online]. Available: https://www.un.org/sustainabledevelopment/health. Accessed on 30/05/2019.

[3]T. W. Bank, "Mortality rate, under-5 (per 1,000 live births)" (n.d)). [Online]. Available: https://data.worldbank.org/indicator/SH.DYN.MORT end $=2017 \&$ locations $=$ MX\&start $=1960 \&$ view $=$ chart. Accessed on 30/05/2019.

[4]T. W. Bank, "Probability of dying at age 5-14 years (per 1,000 children age 5)" (n.d). [Online]. Available: https://datos.bancomundial.org/indicador/SH.DYN.0514. Accessed on 30/05/2019.

[5]Mexican Ministry of Health, "Datos abiertos (in spanish)" (n.d). [Online]. Available: http://www.dgis.salud.gob.mx/contenidos/basesdedatos/Datos_Abiertos_gobmx.html. Accessed on 01/06/2019. 
[6]E. Gayawan, M. I. Adarabioyo, D. M. Okewole, S. G. Fashoto, and J. C. Ukaegbu, “Geographical variations in infant and child mortality in West Africa: A geo-additive discrete-time survival modelling" Genus, vol. 72, no. 1, 2016.

[7]E. Jimenez-soto, J. Durham, and A. Hodge, "Entrenched Geographical and Socioeconomic Disparities in Child Mortality: Trends in Absolute and Relative Inequalities in Cambodia" PLoS One, vol. 9, no. 10, 2014.

[8]A. E. Bauze et al., "Equity and Geography: The Case of Child Mortality in Papua New Guinea", PLoS One, vol. 7, no. 5, 2015.

[9]A. Singh, P. K. Pathak, R. K. Chauhan, and W. Pan, "Infant and Child Mortality in India in the Last Two Decades: A Geospatial Analysis", PLoS One, vol. 6, no. 11, 2011.

[10]A. W. J. Alonso et al., "Spatio-temporal patterns of diarrhoeal mortality in Mexico" Epidemiology and Infection, vol. 140, no. 1, pp. 91-99, 2019.

[11]L. Knorr-Held and J. Besag, "Modelling risk from a disease in time and space" Stat. Med., vol. 17, no. 18, pp. 2045-2060, 1998.

[12]A. L. Papoila et al., "Stomach cancer incidence in Southern Portugal 1998-2006: A spatio-temporal analysis" Biometrical J., vol. 56, no. 3, pp. 403-415, 2014.

[13]A. B. Lawson, Bayesian disease mapping: hierarchical modeling in spatial epidemiology. Chapman and Hall/CRC, 2013.

[14]H. H. Shin, D. Stieb, R. Burnett, G. Takahara, and B. Jessiman, "Tracking National and Regional Spatial-Temporal Mortality Risk Associated with NO2 Concentrations in Canada: A Bayesian Hierarchical Two-Level Model" Risk Anal. An Int. J., vol. 32, no. 3, pp. 513-530, 2012.

[15]M. D. Ugarte, A. Adin, T. Goicoa, I. Casado, E. Ardanaz, and N. Larrañaga, "Temporal evolution of brain cancer incidence in the municipalities of Navarre and the Basque Country, Spain" BMC Public Health, vol. 15, no. 1, pp. 1-7, 2015.

[16]G. Smith, "Area-based initiatives: the rationale and options for area targeting" LSE STICERD Res. Pap. No. Case025, 1999.

[17]R. Tunstall and R. Lupton, "Is Targeting Deprived Areas an Effective Means to Reach Poor People? An assessment of one rationale for area-based funding programmes" 2003.

[18]L. Bernardinelli, D. Clayton, C. Pascutto, C. Montomoli, M. Ghislandi, and M. Songini, "Bayesian analysis of space-time variation in disease risk" Stat. Med., vol. 14, no. 21-22, pp. 2433-2443, 1995.

[19]G. Li, R. Haining, S. Richardson, and N. Best, "Space-time variability in burglary risk: A Bayesian spatio-temporal modelling approach" Spat. Stat., vol. 9, no. C, pp. 180-191, 2014. 
[20]O. for E. C. and D. (OECD), "Territorial Reviews: Valle de México, Mexico," 2015. [Online]. Available: http://www.keepeek.com/Digital Asset-Management/oecd/urban-rural-and-regional-development/oecdterritorial-reviews-valle-de-mexico-mexico/urban-trends-and-challenges-of-the-valle-demexico_9789264245174-5-en. Accessed on 7/06/2019.

[21]M. N. I. of S. and G. (INEGI), "Población,” (n.d). [Online]. Available: https://www.inegi.org.mx/temas/estructura/. Accessed on 7/06/2019.

[22]U. F. and D. Administration, "General clinical pharmacology considerations for pediatric studies for drugs and biological products. Clinical Pharmacology," 2014. [Online]. Available: https://www.fda.gov/Drugs/GuidanceComplianceRegulatorylnformation/Guidances/default.htm. Accessed on 7/06/2019.

[23]L. Anselin, A. K. Bera, R. Florax, and M. J. Yoon, "Simple diagnostic tests for spatial dependence" Reg. Sci. Urban Econ., vol. 26, no. 1, pp. 77-104, 1996.

[24]P. McCullagh, Generalized linear models. Routledge, 2019.

[25]J. Law, M. Quick, and P. Chan, "Bayesian spatio-temporal modeling for analysing local patterns of crime over time at the small-area level” J. Quant. Criminol., vol. 30, no. 1, pp. 57-78, 2014.

[26]J. Besag, J. York, and A. Mollié, "A Bayesian image restoration with two applications in spatial statistics Ann Inst Statist Math 43: 1-59" Find this Artic. online, vol. 43, no. 1, pp. 1-20, 1991.

[27]S. Richardson, A. Thomson, N. Best, and P. Elliott, "Interpreting posterior relative risk estimates in disease-mapping studies" Environ. Health Perspect., vol. 112, no. 9, pp. 1016-1025, 2004.

[28]A. Gelman and D. B. Rubin, "Inference from iterative simulation using multiple sequences" Stat. Sci., vol. 7, no. 4, pp. 457-472, 1992.

[29]C. T. Sreeramareddy, H. N. Harsha Kumar, and B. Sathian, "Time trends and inequalities of under-five mortality in Nepal: A secondary data analysis of four demographic and health surveys between 1996 and 2011" PLoS One, vol. 8, no. 11, pp. 1-12, 2013.

[30]J. M. K. Aheto, "Predictive model and determinants of under-five child mortality: Evidence from the 2014 Ghana demographic and health survey" BMC Public Health, vol. 19, no. 1, pp. 1-10, 2019.

[31]M. of H. in Mexico, "Program de Accion Especifico. Salud Maternal y Perinatal" 2014. [Online]. Available: https://www.gob.mx/cms/uploads/attachment/file/242370. Accessed on 7/06/2019.

[32]S. Havard, S. Deguen, D. Zmirou-Navier, C. Schillinger, and D. Bard, "Traffic-related air pollution and socioeconomic status: a spatial autocorrelation study to assess environmental equity on a small-area scale" Epidemiology, pp. 223-230, 2009. 
[33]D. Fecht et al., "Associations between air pollution and socioeconomic characteristics, ethnicity and age profile of neighbourhoods in England and the Netherlands" Environ. Pollut., vol. 198, pp. 201-210, 2015.

[34] Lome-Hurtado A, Touza-Montero J, White PC. Environmental Injustice in Mexico City: A Spatial Quantile Approach. Exposure and Health, 1-5, 2019.

[35]L. M. McLaughlin, S. D. Johnson, K. J. Bowers, D. J. Birks, and K. Pease, "Police perceptions of the long-and short-term spatial distribution of residential burglary" Int. J. Police Sci. Manag., vol. 9, no. 2, pp. 99-111, 2007.

[36]N. Aragonés et al., "Spatio-temporal trends in gastric cancer mortality in Spain: 1975-2008" Cancer Epidemiol., vol. 37, no. 4, pp. 360-369, 2013.

\section{Table 1}

Descriptive statistical of the observed children mortality risk per 100 inhabitants in Greater Mexico City (from 2012 to 2017).

\begin{tabular}{|c|c|c|c|c|c|c|c|}
\hline $\begin{array}{l}\text { Observed children mortality } \\
\text { risk per } 100 \text { inhabitants }\end{array}$ & $\begin{array}{l}2011 \\
\text { year }\end{array}$ & $\begin{array}{l}2012 \\
\text { year }\end{array}$ & $\begin{array}{l}2013 \\
\text { year }\end{array}$ & $\begin{array}{l}2014 \\
\text { year }\end{array}$ & $\begin{array}{l}2015 \\
\text { year }\end{array}$ & 2016 year & $\begin{array}{l}2017 \\
\text { year }\end{array}$ \\
\hline Mean & 8.17 & 7.73 & 6.85 & 6.54 & 6.15 & 5.21 & 5.41 \\
\hline Standard deviation & 3.15 & 3.03 & 2.52 & 3.08 & 2.58 & 2.16 & 2.23 \\
\hline Minimum value & 1.58 & 1.41 & 0 & 1.63 & 1.49 & 1.3 & 1.33 \\
\hline Maximum value & 17.65 & 15.79 & 12.28 & 15.79 & 14.55 & 13.21 & 10.37 \\
\hline
\end{tabular}

\section{Figures}



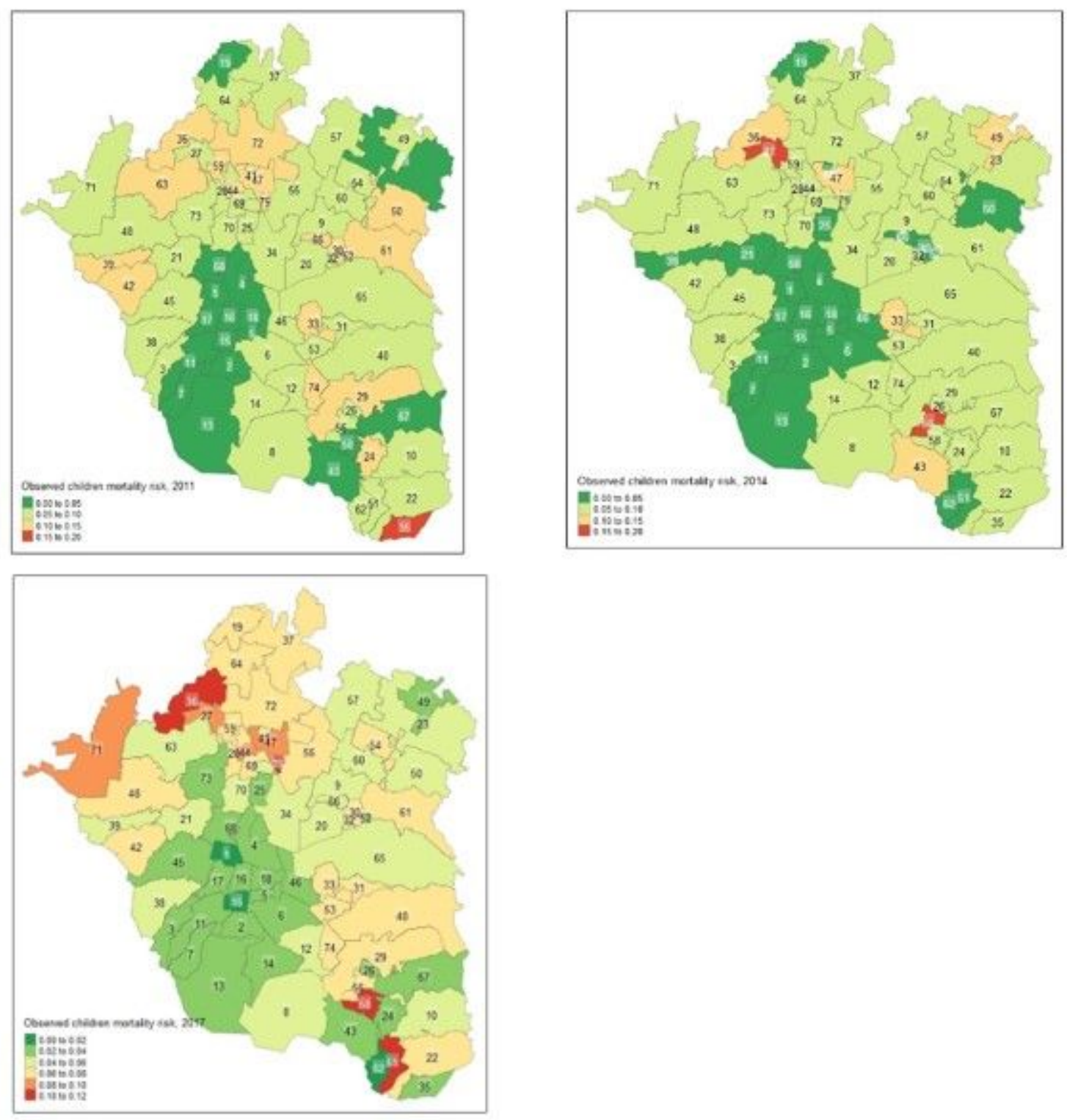

Figure 1

Temporal evolution of the geographical pattern of the observed children mortality risk in Greater Mexico City, at the start, middle, and end $(2011,2014$, and 2017) of the study period. 

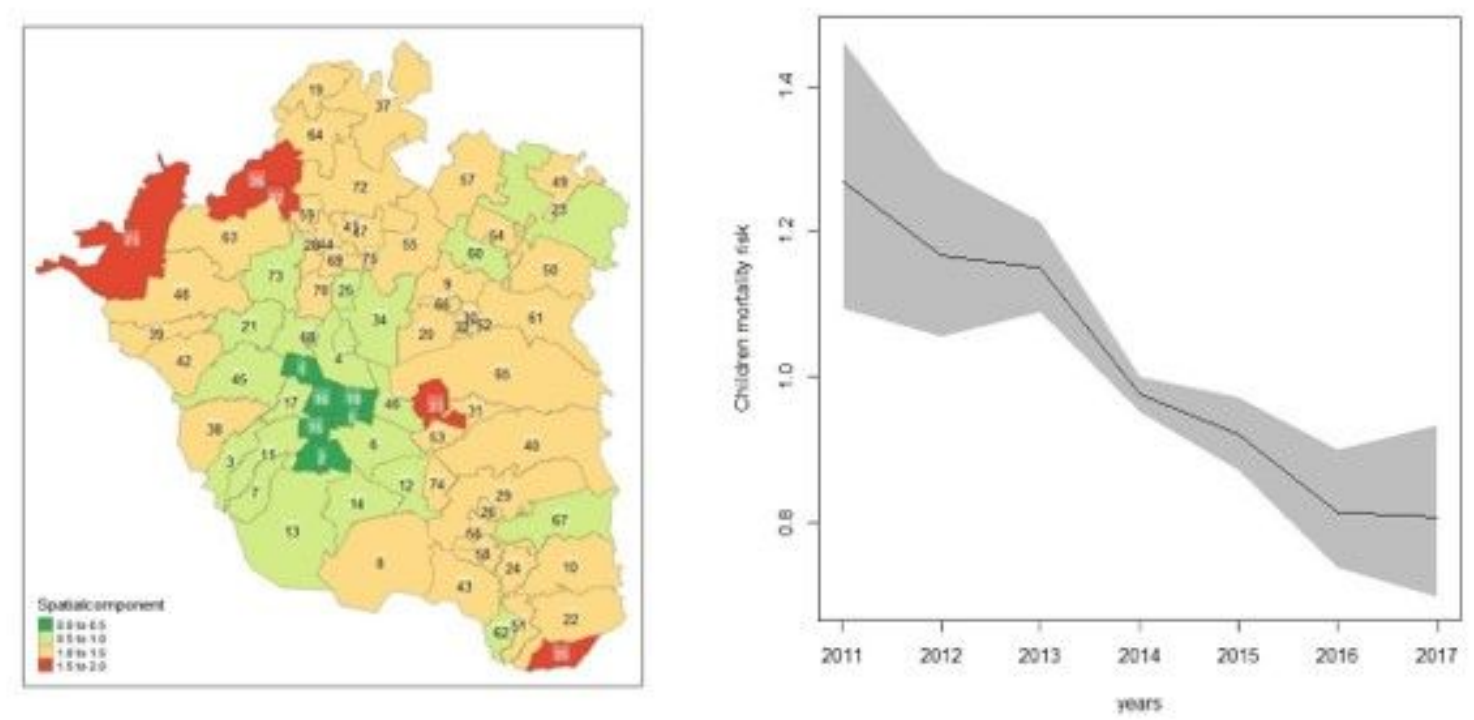

Figure 2

2a. shows the spatial component of children mortality risk odd risk during the studied period. Those areas with an odd risk value greater or lower than 1 have a higher or a lower odd risk respectively, compared with the average. Figure $2 \mathrm{~b}$ displays the overall time trend, in odd risk with $95 \% \mathrm{Cl}$, from 2011 to 2017.
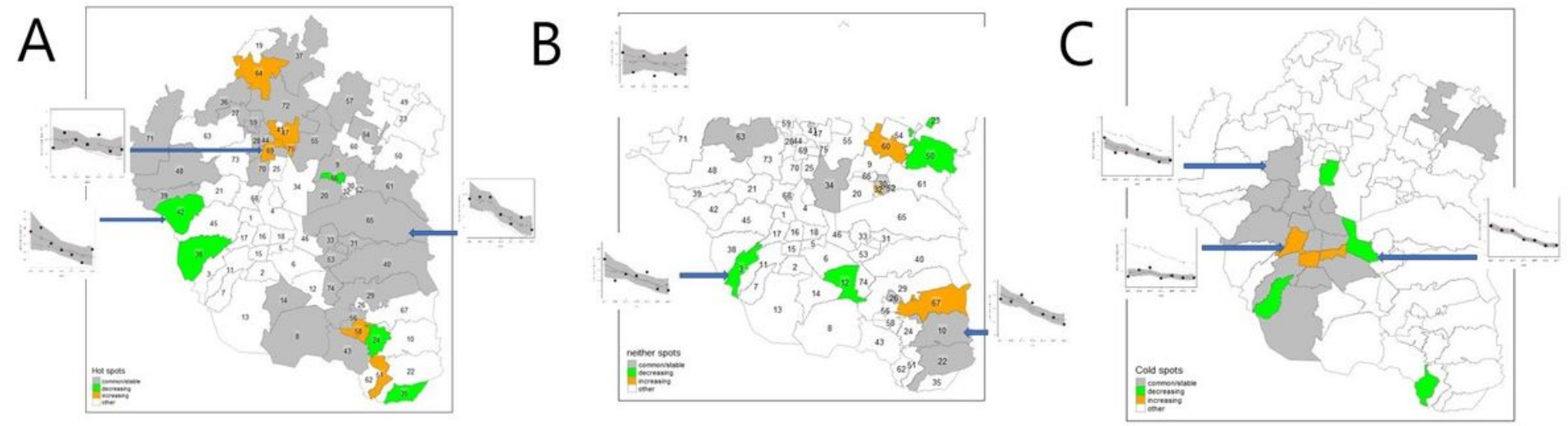

\section{Figure 3}

3a displays the temporal dynamics of children mortality risk for high-risk municipalities in greater Mexico City, which are classified into 3 categories: stable, decreasing and increasing risk. The inserted figures show the observed children mortality risk (per 100 inhabitants; the black solid dots), the estimated children mortality risks -posterior means of risks-(open circles and dashed line) with $95 \% \mathrm{Cl}$ (grey region) and the estimated common trend (black line) over time. $3 \mathrm{~b}$ displays the temporal dynamics of children mortality risk for medium-risk municipalities in greater Mexico City, which are classified into 3 categories: stable, decreasing and increasing risk. The inserted figures show the observed children mortality risk (per 100 inhabitants; the black solid dots), the estimated children mortality risks -posterior means of risks(open circles and dashed line) with $95 \% \mathrm{Cl}$ (grey region) and the estimated common trend (black line) over time. $3 \mathrm{c}$ displays the temporal dynamics of children mortality risk for low-risk municipalities in 
greater Mexico City, which are classified into 3 categories: stable, decreasing and increasing risk. The inserted figures show the observed children mortality risk (per 100 inhabitants; the black solid dots), the estimated children mortality risks -posterior means of risks-(open circles and dashed line) with $95 \% \mathrm{Cl}$ (grey region) and the estimated common trend (black line) over time.

\section{Supplementary Files}

This is a list of supplementary files associated with this preprint. Click to download.

- supplement1.docx 